\title{
Article \\ Proteinuria Indicates Decreased Normal Glomeruli in ANCA-Associated Glomerulonephritis Independent of Systemic Disease Activity
}

\author{
Désirée Tampe $^{1 \oplus}$, Peter Korsten ${ }^{1}\left(\mathbb{D}\right.$, Philipp Ströbel $^{2}$, Samy Hakroush ${ }^{2}\left(\mathbb{D}\right.$ and Björn Tampe ${ }^{1, *} \mathbb{C}$ \\ 1 Department of Nephrology and Rheumatology, University Medical Center Göttingen, \\ 37075 Göttingen, Germany; desiree.tampe@med.uni-goettingen.de (D.T.); \\ peter.korsten@med.uni-goettingen.de (P.K.) \\ 2 Institute of Pathology, University Medical Center Göttingen, 37075 Göttingen, Germany; \\ philipp.stroebel@med.uni-goettingen.de (P.S.); samy.hakroush@med.uni-goettingen.de (S.H.) \\ * Correspondence: bjoern.tampe@med.uni-goettingen.de; Tel.: +49-551-3910575
}

Citation: Tampe, D.; Korsten, P.; Ströbel, P.; Hakroush, S.; Tampe, B. Proteinuria Indicates Decreased Normal Glomeruli in ANCAAssociated Glomerulonephritis Independent of Systemic Disease Activity. J. Clin. Med. 2021, 10, 1538. https://doi.org/10.3390/jcm10071538

Academic Editor: Marc Hilhorst

Received: 23 March 2021

Accepted: 2 April 2021

Published: 6 April 2021

Publisher's Note: MDPI stays neutral with regard to jurisdictional claims in published maps and institutional affiliations.

Copyright: () 2021 by the authors. Licensee MDPI, Basel, Switzerland. This article is an open access article distributed under the terms and conditions of the Creative Commons Attribution (CC BY) license (https:// creativecommons.org/licenses/by/ $4.0 /)$.

\begin{abstract}
Background: Renal involvement is a common and severe complication of antineutrophil cytoplasmic antibody (ANCA)-associated vasculitis (AAV), potentially resulting in a pauci-immune necrotizing and crescentic ANCA glomerulonephritis (GN) with acute kidney injury (AKI), endstage renal disease (ESRD) or death. There is recent evidence that the degree of proteinuria at diagnosis is associated with long-term renal outcome in ANCA GN. Therefore, we here aimed to systematically describe the association between proteinuria and clinicopathological characteristics in 53 renal biopsies with ANCA GN and corresponding urinary samples at admission. Methods: A total number of 53 urinary samples at admission and corresponding renal biopsies with confirmed renal involvement of AAV were retrospectively included from 2015 to 2021 in a single-center study. Results: Proteinuria correlated with myeloperoxidase (MPO) subtype, diagnosis of microscopic polyangiitis (MPA) and severe deterioration of kidney function. Proteinuria was most prominent in sclerotic class ANCA GN and ANCA renal risk score (ARRS) high risk attributed to nonselective proteinuria, including both glomerular and tubular proteinuria. Finally, there was no association between proteinuria and systemic disease activity, suggesting that proteinuria reflected specific renal involvement in AAV rather that systemic disease activity. Conclusions: In conclusion, proteinuria correlated with distinct clinicopathological characteristics in ANCA GN, mostly attributed to a reduced fraction of normal glomeruli. Furthermore, proteinuria in ANCA GN reflected specific renal involvement in AAV rather than systemic disease activity. Therefore, urinary findings could further improve our understanding of mechanisms promoting kidney injury and progression of ANCA GN.
\end{abstract}

Keywords: small vessel vasculitis; ANCA glomerulonephritis; proteinuria

\section{Introduction}

Antineutrophil cytoplasmic antibody (ANCA)-associated vasculitis (AAV) is a small vessel vasculitis according to the 2012 revised Chapel Hill Consensus Conference Nomenclature of Vasculitides, most frequently presenting as microscopic polyangiitis (MPA) or granulomatosis with polyangiitis (GPA) [1,2]. The most severe form of AAV requiring intensive care presents with severe renal and pulmonary involvement, contributing to mortality [3]. Renal involvement is a common complication of AAV, potentially resulting in a pauci-immune necrotizing and crescentic ANCA glomerulonephritis (GN) with acute kidney injury (AKI), end-stage renal disease (ESRD) or death [2]. Clinicopathologic studies of the European Vasculitis Study Group (EUVAS) could demonstrate that the percentage of normal glomeruli, global glomerular sclerosis and the degree of interstitial fibrosis/tubular atrophy (IF/TA) are important parameters related to renal outcome in necrotizing and crescentic GN [4-7]. Derived from these studies, histopathological subgrouping into four 
classes (focal, crescentic, mixed, and sclerotic) as defined by Berden et al. was shown to predict long-term renal survival rates poorest in the sclerotic class (sclerotic glomeruli above 50\%) [8]. These results were confirmed in multiple independent studies over the past years [9-25]. However, multivariable analyses demonstrated no improvement of outcome prediction in most of these studies, mainly attributed to no outcome difference in the crescentic and mixed classes [17-28]. Therefore, Brix et al. suggested the ANCA renal risk score (ARRS), by incorporation of combined IF/TA to the percentage of normal glomeruli and baseline glomerular filtration rate (GFR), to predict ESRD in patients with AAV [29].

The aforementioned previous studies mainly focused on deterioration of kidney function in combination with histopathological findings in ANCA GN. However, there is recent evidence that the degree of proteinuria at diagnosis is associated with long-term renal outcome in ANCA GN $[25,30,31]$. At disease manifestation, the majority of patients with ANCA GN have proteinuria with variable amounts [18]. Previously, we also reported that proteinuria is observed in a considerable subset of ANCA GN and associated with acute deterioration of kidney function at disease onset, implicating an association with active lesions and ANCA manifestation in the kidney [32]. This is confirmed by recent findings that proteinuria associates with established ANCA scoring systems showed the lowest proteinuria in biopsies categorized as focal class, followed by mixed and crescentic class ANCA GN [33]. However, correlation of proteinuria and its subclassification with regard to histopathological findings in ANCA GN has not been systematically described yet. Therefore, we here aimed to systematically describe the association between proteinuria and clinicopathological characteristics in 53 renal biopsies with ANCA GN and corresponding urinary samples at admission.

\section{Materials and Methods}

\subsection{Study Population}

A total number of 53 renal biopsies with confirmed renal involvement of AAV and 53 corresponding urinary samples primarily admitted to the Department of Nephrology and Rheumatology, University Medical Center Göttingen, Germany were retrospectively included from 2015 to 2021; the patient cohort was in part previously described [32]. The study was conducted according to the guidelines of the Declaration of Helsinki and approved by the Ethics Committee of University Medical Center Göttingen, Germany. Medical records were used to obtain data on age, sex, diagnosis (MPA or GPA) and laboratory results. The estimated glomerular filtration rate (GFR) was calculated using the Chronic Kidney Disease Epidemiology Collaboration (CKD-EPI) equation [34]. The worst measurement during the initial course of the disease was used to determine the severity of kidney injury. At admission, the Birmingham Vasculitis Activity Score (BVAS) version 3 was calculated as described previously [35]. The BVAS is assessed on a scale of 0 to 63, with a score of 0 indicating the absence of disease activity and higher scores indicating active disease.

\subsection{Urinary Analysis}

Levels of total proteinuria, urinary albumin, immunoglobulin $G(\operatorname{IgG}), \alpha_{1}$-microglobulin and $\alpha_{2}$-macroglobulin were normalized to urinary creatinine concentration to control for variations in urine flow rate [36]. Leukocyturia and hematuria per high-power field (HPF) were semiquantitatively scored negative (0), 2-4/HPF (1), 5-9/HPF (2), 10-20/HPF (3) and $>20 / \mathrm{HPF}$ (4). Acanthocytes were scored for presence/absence.

\subsection{Renal Histopathology}

A pathologist (SH) evaluated all biopsies, independently validated by a second renal pathologist (PS) blinded to clinical data collection and analysis. Within a renal biopsy specimen, each glomerulus was scored separately for the presence of necrosis, crescents and global sclerosis. Consequently, the percentage of glomeruli with any of these features 
was calculated as a fraction of the total number of glomeruli in each renal biopsy. Based on these scorings, histopathological subgroupings according to Berden et al. (focal, crescentic, mixed or sclerotic class) and ARRS according to Brix et al. (low, medium or high risk) were performed $[8,29]$. Apart from these categories, the degree of interstitial fibrosis and tubular atrophy (IF/TA) was quantified. Renal pathologists were blinded to clinical data collection and analysis.

\subsection{Statistical Methods}

Variables were tested for normal distribution using the Shapiro-Wilk test. Nonnormally distributed continuous variables are expressed as median and interquartile range (IQR), categorical variables are presented as frequency and percentage. Statistical comparisons were not formally powered or prespecified. For group comparisons, the Mann-Whitney U-test was used to determine differences in medians. Nonparametric between-group comparisons were performed with Pearson's Chi-square test. Data analyses were performed with GraphPad Prism (version 8.4.3 for MacOS, GraphPad Software, San Diego, California, U.S.). Multiple regression analyses were performed using IBM SPSS Statistics (version 27 for MacOS, IBM Corporation, Armonk, NY, U.S.). A probability (p) value of $<0.05$ was considered statistically significant.

\section{Results}

\subsection{Proteinuric Findings in ANCA GN}

A total number of 53 renal biopsies with confirmed renal involvement of AAV were identified and retrospectively included from 2015 to 2021. Histopathological subgroupings revealed 17/53 (32.1\%) crescentic, 7/53 (13.2\%) mixed, 26/53 (49.1\%) focal and 3/53 (5.7\%) sclerotic class ANCA GN [8]. ARRS was high in 8/53 (15.1\%), intermediate in 23/53 (43.4\%) and low risk class ANCA GN in 22/53 (41.5\%) cases (Figure 1) [29].

The baseline characteristics of the entire cohort are shown in Table 1. In this cohort, 23/53 (43.4) were female and all were Caucasian. The median (IQR) age at diagnosis was 65 (54.5-74.5) years. Disease onset was 18 (7-46) days before admission, kidney biopsy was performed 6 (3-9.5) days after admission to confirm renal involvement of AAV. There were $26 / 53(49.1 \%)$ positive for myeloperoxidase (MPO) and 27/53 (50.9\%) positive for proteinase 3 (PR3) ANCA. There were 27/53 (50.9\%) patients categorized as MPA and the remainder as GPA. The majority of them (84.9\%) had a new diagnosis of AAV. There were $44 / 53$ patients (83\%) with extrarenal manifestation of AAV (31 with lung, 9 with sinus, 12 with joint, 4 with ear, 3 with eye, 6 with peripheral nerve and 8 with skin involvement); $7 / 53$ (13.2\%) had alveolar hemorrhage. The worst median (IQR) eGFR at disease onset was $19(9.7-50.2) \mathrm{mL} / \mathrm{min} / 1.73 \mathrm{~m}^{2}$, and 16 required dialysis within 30 days after admission. Renal involvement of AAV revealed variable protein-to-creatinine ratios (uPCR) ranging from low-range to nephrotic syndromes; however, most patients presented with subnephrotic proteinuria. This was attributed to nonselective proteinuria, including urinary albumin (uACR), IgG, $\alpha_{1}$-microglobulin and $\alpha_{2}$-macroglobulin, confirming that ANCA GN can present with glomerular, tubular or overflow proteinuria [37]. 


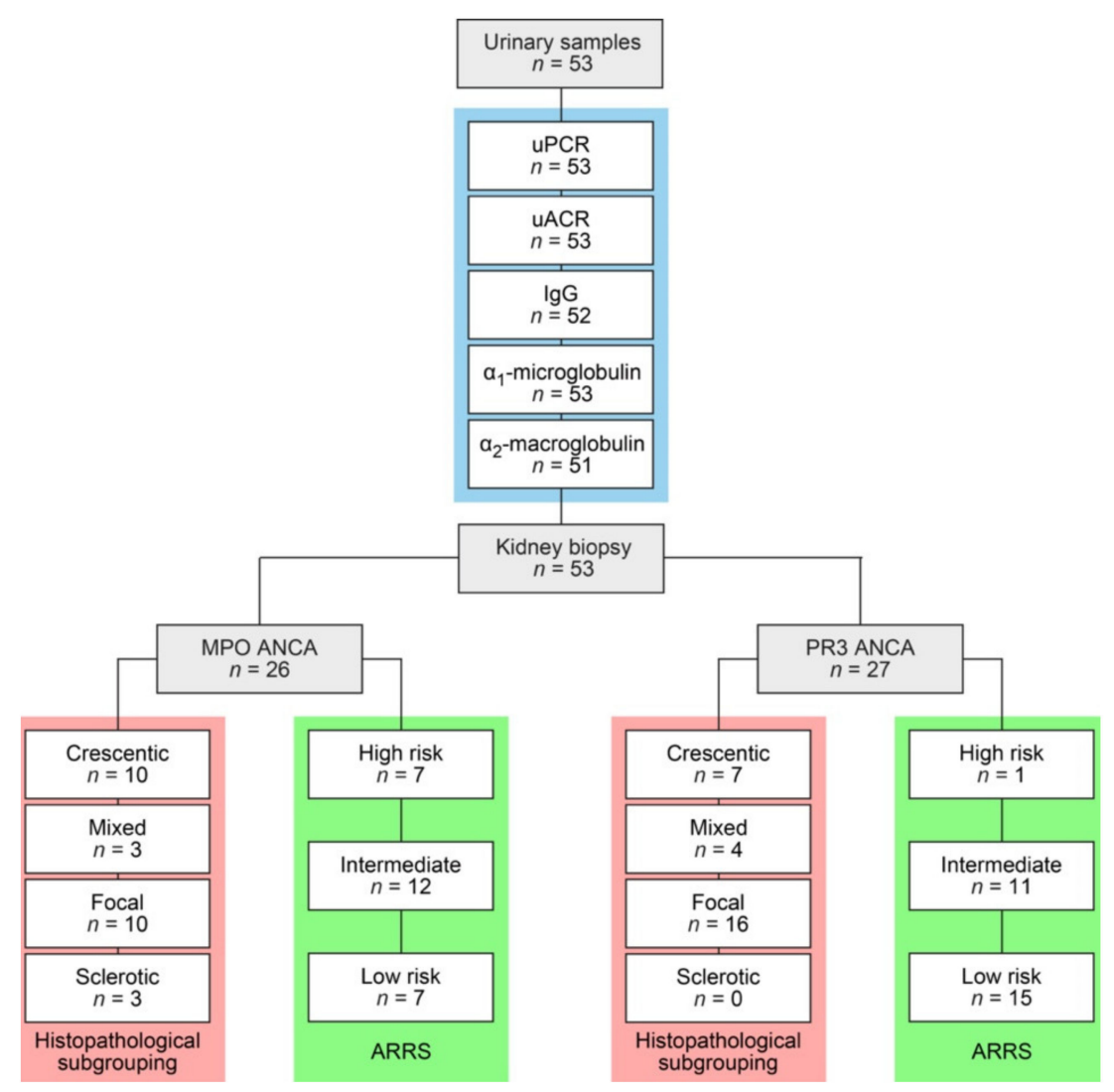

Figure 1. Total patient cohort of ANCA glomerulonephritis (GN). STROBE flow chart of patient disposition. Abbreviations: ANCA, antineutrophil cytoplasmic antibodies; ARRS, ANCA renal risk score; MPO, myeloperoxidase; PR3, proteinase 3; STROBE, Strengthening the Reporting of Observational Studies in Epidemiology; uACR, urinary albumin-to-creatinine ratio; uPCR, urinary protein-to-creatinine ratio.

Table 1. Total patient cohort of ANCA GN.

\begin{tabular}{cc}
\hline Parameter & Value \\
Clinical data & \\
Female sex-no. (\%) & $23(43.4)$ \\
Age (IQR)—years & $65(54.5-74.5)$ \\
Disease onset-days before admission (IQR) & $18(7-46)$ \\
Kidney biopsy-days after admission (IQR) & $6(3-9.5)$ \\
MPO/PR3 subtype-no. (\%) & $26 / 27(49.1 / 50.9)$ \\
MPA/GPA subtype-no. (\%) & $26 / 27(49.1 / 50.9)$ \\
New diagnosis-no. (\%) & $45(84.9)$ \\
\hline Systemic disease activity & \\
BVAS (IQR)-points & $18(15-20.5)$ \\
Extrarenal manifestation-no. (\%) & $44(83)$ \\
Lung involvement-no. (\%) & $31(58.5)$ \\
Alveolar hemorrhage-no. (\%) & $7(13.2)$ \\
Sinus involvement-no. (\%) & $9(17)$ \\
Joint involvement-no. (\%) & $12(22.6)$ \\
Ear involvement-no. (\%) & $4(7.5)$ \\
Eye involvement-no. (\%) & $3(5.7)$ \\
Peripheral nerve involvement-no. (\%) & $6(11.3)$ \\
Skin involvement-no. (\%) & $8(15.1)$ \\
CRP (IQR)-mg/L & $57.4(19.1-107)$ \\
\hline
\end{tabular}


Table 1. Cont.

\begin{tabular}{|c|c|}
\hline Parameter & Value \\
\hline \multicolumn{2}{|l|}{ Renal injury } \\
\hline Serum creatinine (IQR)- $-\mu \mathrm{mol} / \mathrm{L}$ & $269(116-437)$ \\
\hline $\mathrm{eGFR}(\mathrm{IQR})-\mathrm{mL} / \mathrm{min} / 1.73 \mathrm{~m}^{2}$ & $19(9.7-50.2)$ \\
\hline Dialysis within 30 days after admission-no. (\%) & $16(30.2)$ \\
\hline \multicolumn{2}{|l|}{ Proteinuric findings } \\
\hline Leukocyturia (IQR)—per HPF & $2(1-4)$ \\
\hline Hematuria (IQR)—per HPF & $4(3-4)$ \\
\hline Acanthocytes-no. (\%) & $8(15.1)$ \\
\hline $\mathrm{uPCR}(\mathrm{IQR})-\mathrm{mg} / \mathrm{g}$ & $904(505-1653)$ \\
\hline $\mathrm{uACR}(\mathrm{IQR})-\mathrm{mg} / \mathrm{g}$ & $445(164-855)$ \\
\hline IgG/creatinine $(\mathrm{IQR})-\mathrm{mg} / \mathrm{g}$ & $44.1(20.5-191)$ \\
\hline$\alpha_{1}$-microglobulin/creatinine (IQR)—mg/g & $69.6(34.8-172)$ \\
\hline$\alpha_{2}$-macroglobulin/creatinine (IQR)-mg/g & $5.05(2.92-11.1)$ \\
\hline \multicolumn{2}{|l|}{ Histopathological findings } \\
\hline Total glomeruli (IQR)—no. & $17(11-28)$ \\
\hline Normal glomeruli (IQR)—\% & $48.9(26.2-73)$ \\
\hline Glomerular necrosis (IQR)—\% & $15.2(0-44.7)$ \\
\hline Glomerular crescents (IQR)—\% & $30.8(9.76-55.1)$ \\
\hline Glomerular sclerosis (IQR)—\% & $5.13(0-26.2)$ \\
\hline $\mathrm{IF} / \mathrm{TA}(\mathrm{IQR})-\%$ & $20(10-40)$ \\
\hline \multicolumn{2}{|c|}{$\begin{array}{l}\text { Median values and IQR are shown. Hematuria per HPF: negative }=0,2-4=1,5-9=2,10-20=3,>20=4 \text {. } \\
\text { Abbreviations: ANCA, antineutrophil cytoplasmic antibodies; ARRS, ANCA renal risk score; BVAS, Birmingham } \\
\text { Vasculitis Activity Score; CRP, C-reactive protein; eGFR, estimated glomerular filtration rate (CKD-EPI); GN, } \\
\text { glomerulonephritis; HPF, high-power field; IF/TA, interstitial fibrosis/tubular atrophy; IgG, immunoglobulin G; } \\
\text { IQR, interquartile range; MPO, myeloperoxidase; No., number; PR3, proteinase 3; uACR, urinary albumin-to- } \\
\text { creatinine ratio; uPCR, urinary protein-to-creatinine ratio. }\end{array}$} \\
\hline
\end{tabular}

Association with clinical and laboratory findings revealed that uPCR correlated with MPO subtype, diagnosis of MPA and severe deterioration of kidney function assessed by eGFR loss (Figure 2), in line with our previous observations [32]. Proteinuria subclassification established that higher levels of proteinuria in MPO subtype was attributed to increased $\mathrm{uACR}$ and urinary IgG levels (Figure 2), reflecting nonselective glomerular proteinuria. Association between severe deterioration of kidney function was observed for uACR, IgG, $\alpha_{1}$-microglobulin and $\alpha_{1}$-macroglobulin (Figure 2). Interestingly, increased levels of C-reactive protein (CRP) were specifically associated with $\alpha_{1}$-microglobulin (Figure 2), confirming previous observations that urinary $\alpha_{1}$-microglobulin reflects systemic inflammation [38]. In addition, leukocyturia was more frequently observed in patients with relapsing ANCA GN (Figure 2). In summary, ANCA GN presents with subnephrotic proteinuria in most cases, including glomerular and/or tubular proteinuria. Furthermore, proteinuria correlated with more severe deterioration of kidney function in ANCA GN. Therefore, we next aimed to correlate proteinuric findings in association with histopathological findings in ANCA GN.

\subsection{Proteinuric Findings in Correlation with Histopathological Findings in ANCA GN}

By applying scoring of ANCA GN according to Berden et al., highest levels of proteinuria (uPCR) were observed in sclerotic and lowest in focal class ANCA GN (Table 2), in line with previous observations $[8,33]$. Comparable results were also observed for proteinuria subclassification; uACR, IgG, $\alpha_{1}$-microglobulin and $\alpha_{2}$-macroglobulin were most prominent in sclerotic and lowest in focal class ANCA GN (Table 2). Categorization of ANCA GN in ARRS revealed that increased risk class was associated with higher levels of uPCR, equally reflected by proteinuria subclassification in $\mathrm{uACR}, \mathrm{IgG}, \alpha_{1}$-microglobulin and $\alpha_{2}$-macroglobulin (Table 3). 


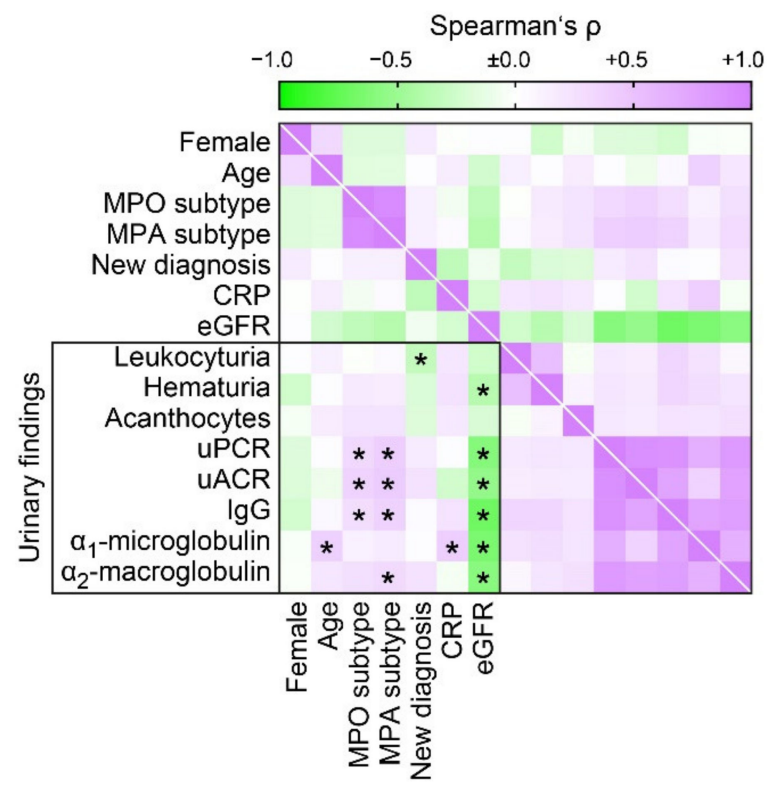

Figure 2. Proteinuric findings in correlation with clinical and laboratory findings in ANCA GN. Association between proteinuria, clinical and laboratory findings is shown by heatmap reflecting mean values of Spearman's $\rho$, asterisks $\left(^{*}\right)$ indicate $p<0.05$. Abbreviations: ANCA, antineutrophil cytoplasmic antibodies; CRP, C-reactive protein; eGFR, estimated glomerular filtration rate (CKD-EPI); GN, glomerulonephritis; IgG, immunoglobulin G; MPA, microscopic polyangiitis; MPO, myeloperoxidase; $\mathrm{uACR}$, urinary albumin-to-creatinine ratio; $\mathrm{uPCR}$, urinary protein-to-creatinine ratio.

Table 2. Proteinuric findings in association with histopathological subgrouping in ANCA GN.

\begin{tabular}{ccc}
\hline Berden Classification & Paratemeter & $p$ Value \\
\hline & uPCR & \\
Crescentic class-mg/g & $1348(755-1939)$ & \\
Focal class-mg/g & $573(359-1213)$ & \\
Mixed class-mg/g & $1540(729-2536)$ & 0.0006 \\
Sclerotic class-mg/g & $5318(5285-8129)$ & \\
Crescentic class-mg/g & uACR & \\
Focal class-mg/g & $678(338-1047)$ & 0.0002 \\
Mixed class-mg/g & $202(86.1-504)$ & \\
Sclerotic class-mg/g & $1021(458-1701)$ & \\
& $3604(3043-4429)$ & 0.0018 \\
Crescentic class-mg/g & Urinary IgG & \\
Focal class-mg/g & $89.1(43.2-232)$ & \\
Mixed class-mg/g & $25(11.2-96.4)$ & \\
Sclerotic class-mg/g & $56.5(20.1-149)$ & \\
\hline & $352(271-542)$ & \\
Crescentic class-mg/g & Urinary $\alpha_{1}$-microglobulin & \\
Focal class-mg/g & $101(52.9-197)$ & \\
Mixed class-mg/g & $41.4(17.6-113)$ & \\
Sclerotic class-mg/g & $67.2(7.24-150)$ & \\
\hline & $191(62.2-310)$ & \\
Crescentic class-mg/g & Urinary $\alpha_{2}$-macroglobulin & \\
Focal class-mg/g & $5.44(3.55-9.03)$ & \\
Mixed class-mg/g & $3.13(1.99-6.27)$ & \\
Sclerotic class-mg/g & $10(4.91-14.1)$ & \\
\hline
\end{tabular}

Median values and IQR are shown. Nonparametric Kruskal-Wallis test was applied for group comparisons. Abbreviations: ANCA, antineutrophil cytoplasmic antibodies; GN, glomerulonephritis; IgG, immunoglobulin G; $\mathrm{IQR}$, interquartile range; $\mathrm{uACR}$, urinary albumin-to-creatinine ratio; $\mathrm{uPCR}$, urinary protein-to-creatinine ratio. 
Table 3. Proteinuric findings in association with ARRS in ANCA GN.

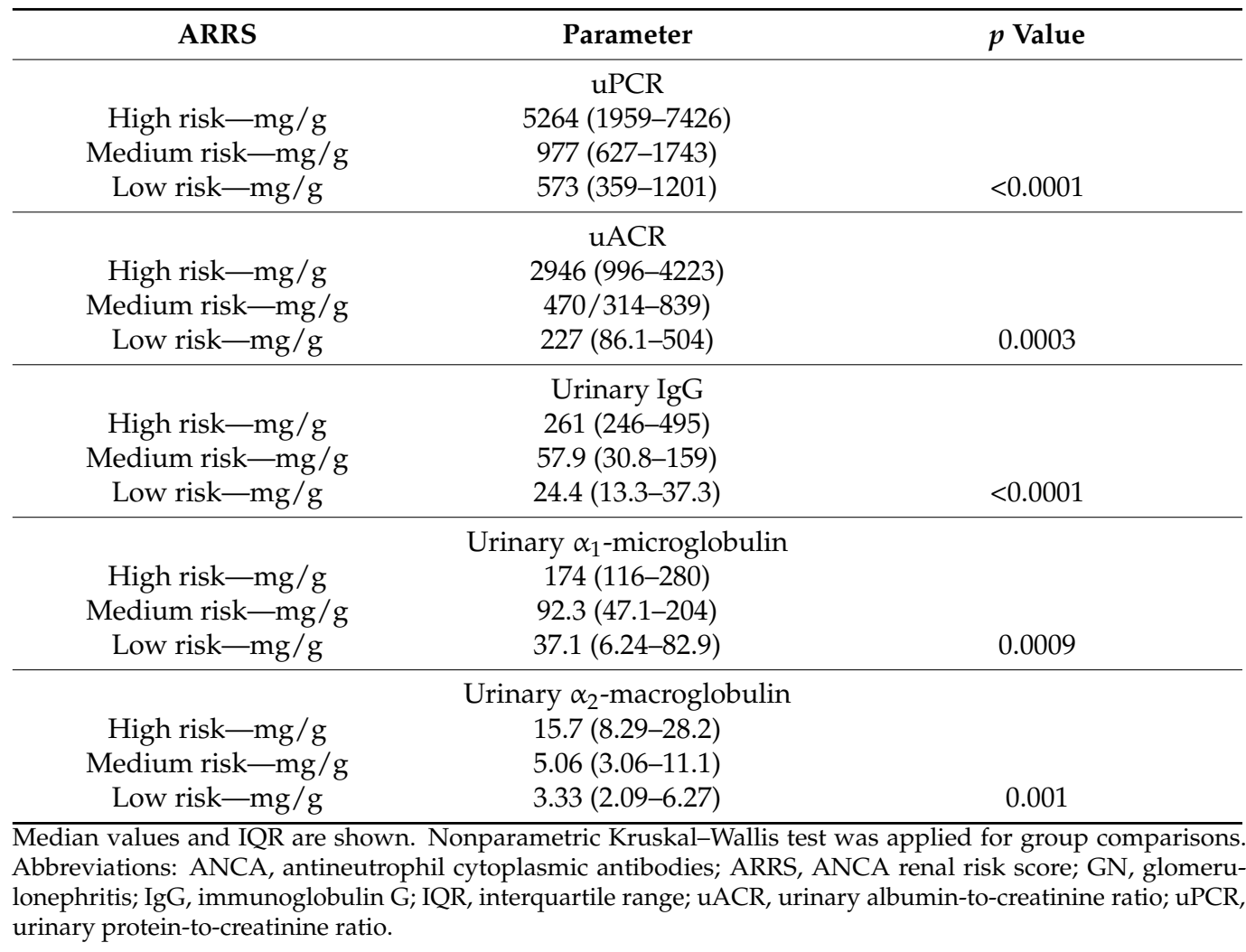

Based on these observations that categorization of ANCA GN into established histopathological scoring systems into sclerotic class ANCA GN and ARRS high risk were associated with highest levels of proteinuria attributed to nonselective proteinuria, we next correlated proteinuric findings in association with distinct histopathological lesions in ANCA GN. Nonselective proteinuria, including uPCR, uACR, urinary IgG, $\alpha_{1}-$ microglobulin and $\alpha_{2}$-macroglobulin was associated with a decreased fraction of normal glomeruli attributed to crescentic ANCA GN (Figure 3A,B). Furthermore, IF/TA also correlated with uPCR, uACR, urinary IgG and $\alpha_{2}$-macroglobulin, but not $\alpha_{1}$-microglobulin (Figure 3B).

Multiple regression analyses revealed a stronger correlation between glomerular proteinuria and a decreased fraction of normal glomeruli as compared to other glomerular lesions or IF/TA in ANCA GN (Table 4), consistent with the concept that each glomerular lesion contributing to a decreased fraction of normal glomeruli needs to be considered [29]. In summary, proteinuria is an independent indicator of decreased normal glomeruli in ANCA GN. Because proteinuria and decreased fraction of normal glomeruli could reflect both specific renal involvement and systemic severity of AAV, we next determined extrarenal manifestation of $\mathrm{AAV}$ in association with proteinuria. 
A

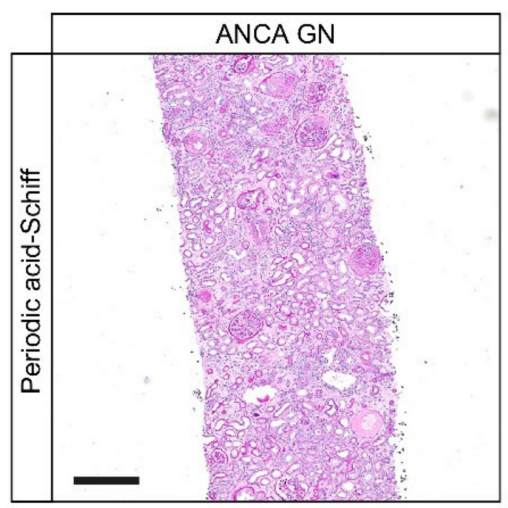

B

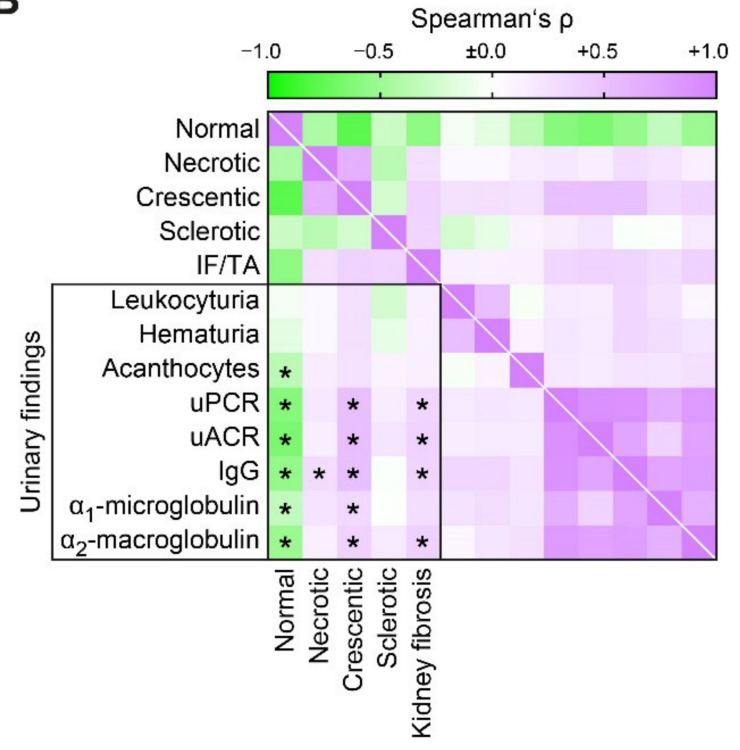

Figure 3. Proteinuric findings in correlation with distinct histopathological findings in ANCA GN. (A) Representative photomicrograph of ANCA GN stained with periodic acid-Schiff (scale bar: $300 \mu \mathrm{m}$ ). (B) Association between proteinuria and histopathological findings is shown by heatmap reflecting mean values of Spearman's $\rho$, asterisks $\left(^{*}\right)$ indicate $p<0.05$. Abbreviations: ANCA, antineutrophil cytoplasmic antibodies; GN, glomerulonephritis; IF/TA, interstitial fibrosis/tubular atrophy; IgG, immunoglobulin G; uACR, urinary albumin-to-creatinine ratio; uPCR, urinary protein-to-creatinine ratio.

Table 4. Multiple regression analysis for variables associated with proteinuria.

\begin{tabular}{|c|c|c|c|}
\hline Variable & $\beta$ & SE & $p$ Value \\
\hline & \multicolumn{3}{|c|}{ uPCR } \\
\hline Normal glomeruli-\% & -47.963 & 14.352 & 0.0016 \\
\hline Glomerular crescents-\% & -18.551 & 12.725 & 0.1513 \\
\hline \multirow[t]{2}{*}{$\mathrm{IF} / \mathrm{TA}-\%$} & 11.895 & 14.609 & 0.4195 \\
\hline & \multicolumn{3}{|c|}{$\mathrm{uACR}$} \\
\hline Normal glomeruli-\% & -33.080 & 9.736 & 0.0014 \\
\hline Glomerular crescents- $\%$ & -10.111 & 8.633 & 0.2472 \\
\hline \multirow[t]{2}{*}{$\mathrm{IF} / \mathrm{TA} — \%$} & 0.456 & 0.911 & 0.9635 \\
\hline & \multicolumn{3}{|c|}{ Urinary IgG } \\
\hline Normal glomeruli—\% & -2.237 & 1.269 & 0.0845 \\
\hline Glomerular necrosis—\% & -1.662 & 1.117 & 0.1435 \\
\hline Glomerular crescents- $\%$ & 0.865 & 1.512 & 0.5697 \\
\hline \multirow[t]{2}{*}{$\mathrm{IF} / \mathrm{TA}-\%$} & 2.032 & 1.117 & 0.1129 \\
\hline & \multicolumn{3}{|c|}{ Urinary $\alpha_{1}$-microglobulin } \\
\hline Normal glomeruli-\% & -0.255 & 1.207 & 0.8336 \\
\hline \multirow[t]{2}{*}{ Glomerular crescents- $\%$} & 0.757 & 1.217 & 0.5367 \\
\hline & \multicolumn{3}{|c|}{ Urinary $\alpha_{2}$-macroglobulin } \\
\hline Normal glomeruli-\% & -0.333 & 0.073 & $<0.0001$ \\
\hline Glomerular crescents- $\%$ & -0.211 & 0.065 & 0.002 \\
\hline $\mathrm{IF} / \mathrm{TA}-\%$ & -0.083 & 0.075 & 0.2726 \\
\hline
\end{tabular}

Abbreviations: ANCA, antineutrophil cytoplasmic antibodies; GN, glomerulonephritis; IgG, immunoglobulin $\mathrm{G}_{;}$ $\mathrm{SE}$, standard error; $\mathrm{uACR}$, urinary albumin-to-creatinine ratio; $\mathrm{uPCR}$, urinary protein-to-creatinine ratio.

\subsection{Proteinuric Findings in Correlation with Extrarenal Manifestations of AAV}

We next examined the correlation between proteinuria and extrarenal manifestation of AAV. We observed no association between proteinuria and systemic disease activity assessed by the BVAS despite previously observed correlation between proteinuria with 
more severe deterioration of kidney function in ANCA GN (Figure 4), implicating that proteinuria associates with decreased extrarenal manifestations of AAV. By systematic analysis of lung, sinus, joint, ear, eye, peripheral nerve, skin involvement or alveolar hemorrhage, we confirmed less involvement of sinus and joint in association with proteinuria (Figure 4). In summary, these observations suggested that proteinuria reflected specific renal involvement in $\mathrm{AAV}$ rather that systemic disease activity.

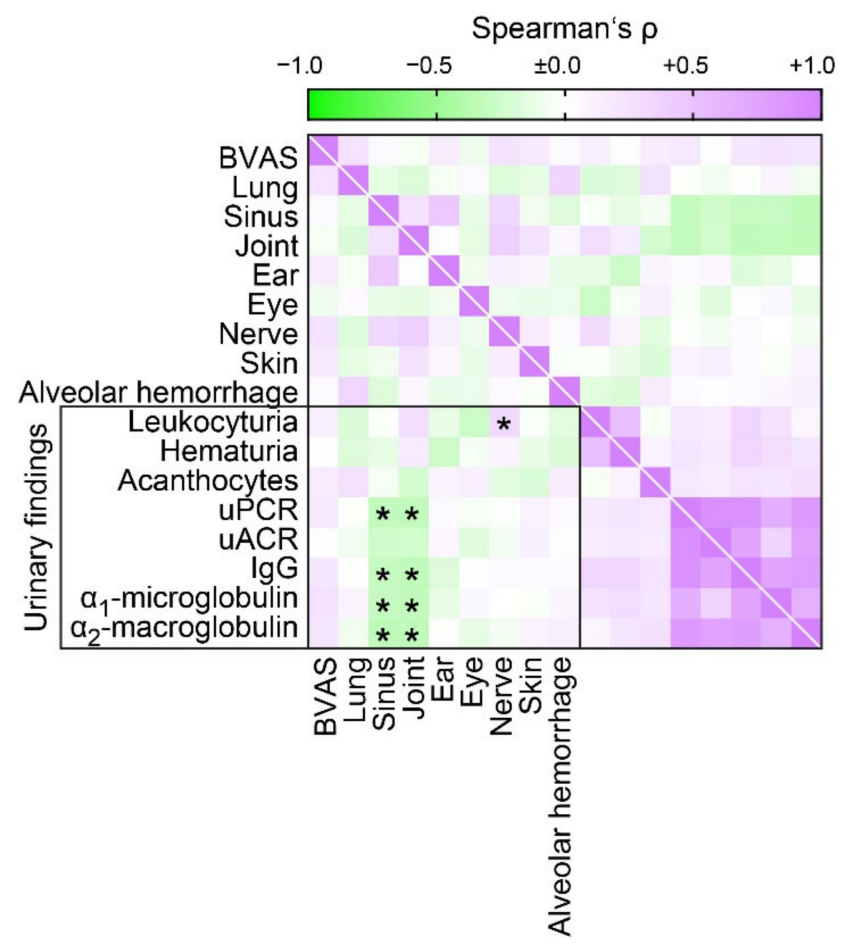

Figure 4. Proteinuric findings in correlation with extrarenal manifestations in AAV. Association between proteinuria and extrarenal manifestations is shown by heatmap reflecting mean values of Spearman's $\rho$, asterisks $\left(^{*}\right)$ indicate $p<0.05$. Abbreviations: ANCA, antineutrophil cytoplasmic antibodies; BVAS, Birmingham Vasculitis Activity Score; GN, glomerulonephritis; IgG, immunoglobulin G; uACR, urinary albumin-to-creatinine ratio; $\mathrm{uPCR}$, urinary protein-to-creatinine ratio.

\section{Discussion}

We here aimed to systematically describe the correlation between urinary findings and clinicopathological characteristics in ANCA GN. Proteinuria correlated with MPO subtype, diagnosis of MPA and severe deterioration of kidney function, in line with our previous observations [32]. Proteinuria subclassification established that higher levels of proteinuria in MPO subtype were attributed to nonselective glomerular proteinuria. At disease manifestation, renal involvement of AAV can either present with active lesions including glomerular crescents and necrosis, or with chronic lesions including global glomerular sclerosis. The pathologic activity and chronicity of ANCA GN can be classified by histopathological subgrouping (crescentic, mixed, focal and sclerotic) or ARRS (high, intermediate and low risk) [8,29]. Aforementioned previous studies have mainly focused on deterioration of kidney function in combination with histopathological findings in ANCA GN. However, there is recent evidence that the degree of proteinuria at diagnosis is associated with long-term renal outcome in ANCA GN [25,30,31]. Proteinuria is the hallmark of GN and the most important predictor of outcome, including diabetes-related and idiopathic glomerular kidney diseases [39-47]. We here show that categorization of ANCA GN into established histopathological scoring systems revealed that sclerotic class ANCA GN and ARRS high risk were associated with highest levels of proteinuria attributed to nonselective proteinuria, including both glomerular and tubular proteinuria. In contrast, lowest proteinuria was observed in focal class ANCA GN, in line with recent findings that 
proteinuria associates with established ANCA scoring systems showing lowest proteinuria in biopsies categorized as focal class, followed by mixed and crescentic class ANCA GN [33]. Multivariate regression analyses revealed that only decreased fraction of normal glomeruli independently associated with proteinuria. It has previously been proposed that damaged glomeruli have the capability to recover and active lesions may be reversible in principle, at least to a certain extent $[5,30]$. In addition, the fraction of normal glomeruli (without scarring, crescents or necrosis within the tuft) was the strongest independent predictor of death-censored ESRD in ANCA GN [29]. Therefore, each glomerular lesion needs to be considered, emphasizing the importance of normal glomeruli and an early diagnosis. Studies of repeat biopsies strengthen this hypothesis since previous studies have demonstrated the progression of active into chronic lesions in ANCA GN over time $[30,48]$. This could in part explain the variable outcomes of patients that are classified as crescentic and mixed in the validation of the classification [9,14,22,24].

Finally, there was no association between proteinuria and systemic disease activity assessed by the BVAS despite previously observed correlation between proteinuria with more severe deterioration of kidney function in ANCA GN. Since the BVAS also includes deterioration of kidney function, these results implicate that proteinuria associates with decreased extrarenal manifestations of AAV. We here provide evidence that proteinuria correlated with less involvement of sinus and joint in AAV. These observations suggested that proteinuria reflected specific renal involvement in AAV rather that systemic disease activity. This interesting phenomenon needs further investigation and has independently been observed [49].

The main limitations of our study are its retrospective design in a single center, a selection bias towards more severe cases of ANCA GN with limited information of kidney function before disease manifestation as a tertiary referral center and lack of long-term renal survival rates. Nevertheless, the number of patients with ANCA GN and severe deterioration of kidney function at our center is considerable.

\section{Conclusions}

In conclusion, proteinuria correlated with distinct clinicopathological characteristics in ANCA GN, mostly attributed to a reduced fraction of normal glomeruli. Furthermore, proteinuria in ANCA GN reflected specific renal involvement in AAV rather than systemic disease activity. Therefore, urinary findings could further improve our understanding of mechanisms promoting kidney injury and progression of ANCA GN.

Author Contributions: B.T. conceived the study, collected and analyzed data and wrote the first draft. D.T. and P.K. collected and analyzed data. P.S. and S.H. evaluated histopathological findings. D.T., P.K., P.S. and S.H. edited the manuscript. All authors have read and agreed to the published version of the manuscript.

Funding: This research was funded by the Research program, University Medical Center, University of Göttingen, grant number 1403720. This research was also funded by the German Research Foundation, KFO (CRU) 5002, grant number STR 638/3-1 (DFG). We also acknowledge support by the Open Access Publication Funds of the Göttingen University.

Institutional Review Board Statement: The study was conducted according to the guidelines of the Declaration of Helsinki and approved by the Ethics Committee of University Medical Center Göttingen (protocol codes: 22/2/14, approval date 22 September 2014 and 28/09/17, approval date 17 November 2017).

Informed Consent Statement: Informed consent was obtained from all subjects involved in the study.

Data Availability Statement: Deidentified data are available on reasonable request from the corresponding author.

Acknowledgments: The authors thank Ulrike Ehbrecht for technical assistance. 
Conflicts of Interest: The authors declare no conflict of interest. The funders had no role in the design of the study; in the collection, analyses, or interpretation of data; in the writing of the manuscript, or in the decision to publish the results.

\section{References}

1. Jennette, J.C.; Falk, R.J.; Bacon, P.A.; Basu, N.; Cid, M.C.; Ferrario, F.; Flores-Suarez, L.F.; Gross, W.L.; Guillevin, L.; Hagen, E.C.; et al. 2012 revised International Chapel Hill Consensus Conference Nomenclature of Vasculitides. Arthritis Rheum. 2013, 65, 1-11. [CrossRef] [PubMed]

2. Hruskova, Z.; Stel, V.S.; Jayne, D.; Aasarod, K.; De Meester, J.; Ekstrand, A.; Eller, K.; Heaf, J.G.; Hoitsma, A.; Martos Jimenez, C.; et al. Characteristics and Outcomes of Granulomatosis With Polyangiitis (Wegener) and Microscopic Polyangiitis Requiring Renal Replacement Therapy: Results From the European Renal Association-European Dialysis and Transplant Association Registry. Am. J. Kidney Dis. 2015, 66, 613-620. [CrossRef] [PubMed]

3. Demiselle, J.; Auchabie, J.; Beloncle, F.; Gatault, P.; Grange, S.; Du Cheyron, D.; Dellamonica, J.; Boyer, S.; Beauport, D.T.; Piquilloud, L.; et al. Patients with ANCA-associated vasculitis admitted to the intensive care unit with acute vasculitis manifestations: A retrospective and comparative multicentric study. Ann. Intensive Care 2017, 7, 39. [CrossRef] [PubMed]

4. Bajema, I.M.; Hagen, E.C.; Hermans, J.; Noel, L.H.; Waldherr, R.; Ferrario, F.; Van Der Woude, F.J.; Bruijn, J.A. Kidney biopsy as a predictor for renal outcome in ANCA-associated necrotizing glomerulonephritis. Kidney Int. 1999, 56, 1751-1758. [CrossRef] [PubMed]

5. Hauer, H.A.; Bajema, I.M.; Van Houwelingen, H.C.; Ferrario, F.; Noel, L.H.; Waldherr, R.; Jayne, D.R.; Rasmussen, N.; Bruijn, J.A.; Hagen, E.C.; et al. Determinants of outcome in ANCA-associated glomerulonephritis: A prospective clinico-histopathological analysis of 96 patients. Kidney Int. 2002, 62, 1732-1742. [CrossRef]

6. Van Wijngaarden, R.A.D.L.; Hauer, H.A.; Wolterbeek, R.; Jayne, D.R.; Gaskin, G.; Rasmussen, N.; Noel, L.H.; Ferrario, F.; Waldherr R.; Hagen, E.C.; et al. Clinical and histologic determinants of renal outcome in ANCA-associated vasculitis: A prospective analysis of 100 patients with severe renal involvement. J. Am. Soc. Nephrol. 2006, 17, 2264-2274. [CrossRef]

7. Bajema, I.M.; Hagen, E.C.; Hansen, B.E.; Hermans, J.; Noel, L.H.; Waldherr, R.; Ferrario, F.; van der Woude, F.J.; Bruijn, J.A. The renal histopathology in systemic vasculitis: An international survey study of inter- and intra-observer agreement. Nephrol. Dial. Transplant. 1996, 11, 1989-1995. [CrossRef]

8. Berden, A.E.; Ferrario, F.; Hagen, E.C.; Jayne, D.R.; Jennette, J.C.; Joh, K.; Neumann, I.; Noel, L.H.; Pusey, C.D.; Waldherr, R.; et al. Histopathologic classification of ANCA-associated glomerulonephritis. J. Am. Soc. Nephrol. 2010, 21, 1628-1636. [CrossRef]

9. Chang, D.Y.; Wu, L.H.; Liu, G.; Chen, M.; Kallenberg, C.G.; Zhao, M.H. Re-evaluation of the histopathologic classification of ANCA-associated glomerulonephritis: A study of 121 patients in a single center. Nephrol. Dial. Transplant. 2012, 27, 2343-2349. [CrossRef] [PubMed]

10. Ellis, C.L.; Manno, R.L.; Havill, J.P.; Racusen, L.C.; Geetha, D. Validation of the new classification of pauci-immune glomerulonephritis in a United States cohort and its correlation with renal outcome. BMC Nephrol. 2013, 14, 210. [CrossRef]

11. Togashi, M.; Komatsuda, A.; Nara, M.; Omokawa, A.; Okuyama, S.; Sawada, K.; Wakui, H. Validation of the 2010 histopathological classification of ANCA-associated glomerulonephritis in a Japanese single-center cohort. Mod. Rheumatol. 2013. [CrossRef] [PubMed]

12. Rahmattulla, C.; Bruijn, J.A.; Bajema, I.M. Histopathological classification of antineutrophil cytoplasmic antibody-associated glomerulonephritis: An update. Curr. Opin. Nephrol. Hypertens. 2014, 23, 224-231. [CrossRef] [PubMed]

13. Lee, T.; Gasim, A.; Derebail, V.K.; Chung, Y.; McGregor, J.G.; Lionaki, S.; Poulton, C.J.; Hogan, S.L.; Jennette, J.C.; Falk, R.J.; et al. Predictors of treatment outcomes in ANCA-associated vasculitis with severe kidney failure. Clin. J. Am. Soc. Nephrol. 2014, 9 , 905-913. [CrossRef]

14. Diaz-Crespo, F.; Villacorta, J.; Acevedo, M.; Cavero, T.; Guerrero, C.; Garcia Diaz, E.; Orradre, J.L.; Martinez, M.A.; Praga, M.; Fernandez-Juarez, G. The predictive value of kidney biopsy in renal vasculitis: A multicenter cohort study. Hum. Pathol. 2016, 52, 119-127. [CrossRef] [PubMed]

15. Nohr, E.; Girard, L.; James, M.; Benediktsson, H. Validation of a histopathologic classification scheme for antineutrophil cytoplasmic antibody-associated glomerulonephritis. Hum. Pathol. 2014, 45, 1423-1429. [CrossRef] [PubMed]

16. Noone, D.G.; Twilt, M.; Hayes, W.N.; Thorner, P.S.; Benseler, S.; Laxer, R.M.; Parekh, R.S.; Hebert, D. The new histopathologic classification of ANCA-associated GN and its association with renal outcomes in childhood. Clin. J. Am. Soc. Nephrol. 2014, 9 , 1684-1691. [CrossRef]

17. Quintana, L.F.; Perez, N.S.; De Sousa, E.; Rodas, L.M.; Griffiths, M.H.; Sole, M.; Jayne, D. ANCA serotype and histopathological classification for the prediction of renal outcome in ANCA-associated glomerulonephritis. Nephrol. Dial. Transplant. 2014, 29, 1764-1769. [CrossRef] [PubMed]

18. Hilhorst, M.; Wilde, B.; van Breda Vriesman, P.; van Paassen, P.; Cohen Tervaert, J.W.; Limburg Renal, R. Estimating renal survival using the ANCA-associated GN classification. J. Am. Soc. Nephrol. 2013, 24, 1371-1375. [CrossRef]

19. Iwakiri, T.; Fujimoto, S.; Kitagawa, K.; Furuichi, K.; Yamahana, J.; Matsuura, Y.; Yamashita, A.; Uezono, S.; Shimao, Y.; Hisanaga, S.; et al. Validation of a newly proposed histopathological classification in Japanese patients with anti-neutrophil cytoplasmic antibody-associated glomerulonephritis. BMC Nephrol. 2013, 14, 125. [CrossRef] [PubMed] 
20. Ford, S.L.; Polkinghorne, K.R.; Longano, A.; Dowling, J.; Dayan, S.; Kerr, P.G.; Holdsworth, S.R.; Kitching, A.R.; Summers, S.A. Histopathologic and clinical predictors of kidney outcomes in ANCA-associated vasculitis. Am. J. Kidney Dis. 2014, 63, 227-235. [CrossRef]

21. Naidu, G.S.; Sharma, A.; Nada, R.; Kohli, H.S.; Jha, V.; Gupta, K.L.; Sakhuja, V.; Rathi, M. Histopathological classification of pauci-immune glomerulonephritis and its impact on outcome. Rheumatol. Int. 2014, 34, 1721-1727. [CrossRef] [PubMed]

22. Moroni, G.; Binda, V.; Leoni, A.; Raffiotta, F.; Quaglini, S.; Banfi, G.; Messa, P. Predictors of renal survival in ANCA-associated vasculitis. Validation of a histopatological classification schema and review of the literature. Clin. Exp. Rheumatol. 2015, 33, S56-S63.

23. Andreiana, I.; Stancu, S.; Avram, A.; Taran, L.; Mircescu, G. ANCA positive crescentic glomerulonephritis outcome in a Central East European cohort: A retrospective study. BMC Nephrol. 2015, 16, 90. [CrossRef] [PubMed]

24. Tanna, A.; Guarino, L.; Tam, F.W.; Rodriquez-Cubillo, B.; Levy, J.B.; Cairns, T.D.; Griffith, M.; Tarzi, R.M.; Caplin, B.; Salama, A.D.; et al. Long-term outcome of anti-neutrophil cytoplasm antibody-associated glomerulonephritis: Evaluation of the international histological classification and other prognostic factors. Nephrol. Dial. Transplant. 2015, 30, 1185-1192. [CrossRef]

25. Cordova-Sanchez, B.M.; Mejia-Vilet, J.M.; Morales-Buenrostro, L.E.; Loyola-Rodriguez, G.; Uribe-Uribe, N.O.; Correa-Rotter, R. Clinical presentation and outcome prediction of clinical, serological, and histopathological classification schemes in ANCAassociated vasculitis with renal involvement. Clin. Rheumatol. 2016, 35, 1805-1816. [CrossRef]

26. Van Daalen, E.; Ferrario, F.; Noel, L.H.; Waldherr, R.; Hagen, E.C.; Bruijn, J.A.; Bajema, I.M. Twenty-five years of RENHIS: A history of histopathological studies within EUVAS. Nephrol. Dial. Transplant. 2015, 30, i31-i36. [CrossRef]

27. Bjorneklett, R.; Sriskandarajah, S.; Bostad, L. Prognostic Value of Histologic Classification of ANCA-Associated Glomerulonephritis. Clin. J. Am. Soc. Nephrol. 2016, 11, 2159-2167. [CrossRef]

28. Chen, Y.X.; Xu, J.; Pan, X.X.; Shen, P.Y.; Li, X.; Ren, H.; Chen, X.N.; Ni, L.Y.; Zhang, W.; Chen, N. Histopathological Classification and Renal Outcome in Patients with Antineutrophil Cytoplasmic Antibodies-associated Renal Vasculitis: A Study of 186 Patients and Metaanalysis. J. Rheumatol. 2017, 44, 304-313. [CrossRef]

29. Brix, S.R.; Noriega, M.; Tennstedt, P.; Vettorazzi, E.; Busch, M.; Nitschke, M.; Jabs, W.J.; Ozcan, F.; Wendt, R.; Hausberg, M.; et al. Development and validation of a renal risk score in ANCA-associated glomerulonephritis. Kidney Int. 2018, 94, 1177-1188, [CrossRef]

30. Neumann, I.; Kain, R.; Regele, H.; Soleiman, A.; Kandutsch, S.; Meisl, F.T. Histological and clinical predictors of early and late renal outcome in ANCA-associated vasculitis. Nephrol. Dial. Transplant. 2005, 20, 96-104. [CrossRef]

31. Franssen, C.F.; Stegeman, C.A.; Oost-Kort, W.W.; Kallenberg, C.G.; Limburg, P.C.; Tiebosch, A.; De Jong, P.E.; Tervaert, J.W. Determinants of renal outcome in anti-myeloperoxidase-associated necrotizing crescentic glomerulonephritis. J. Am. Soc. Nephrol. 1998, 9, 1915-1923.

32. Hakroush, S.; Tampe, D.; Korsten, P.; Stroebel, P.; Zeisberg, M.; Tampe, B. Histopathological findings predict renal recovery in severe ANCA-associated vasculitis requiring intensive care treatment. Front. Med. 2020, 7, 622028. [CrossRef] [PubMed]

33. Van Daalen, E.E.; Neeskens, P.; Zandbergen, M.; Harper, L.; Karras, A.; Vaglio, A.; de Zoysa, J.; Bruijn, J.A.; Bajema, I.M. Podocytes and Proteinuria in ANCA-Associated Glomerulonephritis: A Case-Control Study. Front. Immunol. 2019, 10, 1405. [CrossRef] [PubMed]

34. Levey, A.S.; Stevens, L.A.; Schmid, C.H.; Zhang, Y.L.; Castro, A.F., 3rd; Feldman, H.I.; Kusek, J.W.; Eggers, P.; Van Lente, F.; Greene, T.; et al. A new equation to estimate glomerular filtration rate. Ann. Intern. Med. 2009, 150, 604-612. [CrossRef]

35. Mukhtyar, C.; Lee, R.; Brown, D.; Carruthers, D.; Dasgupta, B.; Dubey, S.; Flossmann, O.; Hall, C.; Hollywood, J.; Jayne, D.; et al. Modification and validation of the Birmingham Vasculitis Activity Score (version 3). Ann. Rheum. Dis. 2009, 68, 1827-1832. [CrossRef]

36. Waikar, S.S.; Sabbisetti, V.S.; Bonventre, J.V. Normalization of urinary biomarkers to creatinine during changes in glomerular filtration rate. Kidney Int. 2010, 78, 486-494. [CrossRef] [PubMed]

37. Abuelo, J.G. Proteinuria: Diagnostic principles and procedures. Ann. Intern. Med. 1983, 98, 186-191. [CrossRef]

38. Vyssoulis, G.P.; Tousoulis, D.; Antoniades, C.; Dimitrakopoulos, S.; Zervoudaki, A.; Stefanadis, C. Alpha-1 microglobulin as a new inflammatory marker in newly diagnosed hypertensive patients. Am. J. Hypertens. 2007, 20, 1016-1021. [CrossRef]

39. Mogensen, C.E. Progression of nephropathy in long-term diabetics with proteinuria and effect of initial anti-hypertensive treatment. Scand. J. Clin. Lab. Investig. 1976, 36, 383-388. [CrossRef]

40. Row, P.G.; Cameron, J.S.; Turner, D.R.; Evans, D.J.; White, R.H.; Ogg, C.S.; Chantler, C.; Brown, C.B. Membranous nephropathy long-term follow-up and association with neoplasia. QJM Int. J. Med. 1975, 44, 207-239.

41. Cameron, J.S. Proteinuria and progression in human glomerular diseases. Am. J. Nephrol. 1990, 10, 81-87. [CrossRef] [PubMed]

42. Gall, M.A.; Nielsen, F.S.; Smidt, U.M.; Parving, H.H. The course of kidney function in type 2 (non-insulin-dependent) diabetic patients with diabetic nephropathy. Diabetologia 1993, 36, 1071-1078. [CrossRef] [PubMed]

43. Lewis, E.J.; Hunsicker, L.G.; Bain, R.P.; Rohde, R.D. The effect of angiotensin-converting-enzyme inhibition on diabetic nephropathy. The Collaborative Study Group. N. Engl. J. Med. 1993, 329, 1456-1462. [CrossRef] [PubMed]

44. Myers, B.D.; Nelson, R.G.; Tan, M.; Beck, G.J.; Bennett, P.H.; Knowler, W.C.; Blouch, K.; Mitch, W.E. Progression of overt nephropathy in non-insulin-dependent diabetes. Kidney Int. 1995, 47, 1781-1789. [CrossRef]

45. Christensen, P.K.; Rossing, P.; Nielsen, F.S.; Parving, H.H. Natural course of kidney function in Type 2 diabetic patients with diabetic nephropathy. Diabet. Med. 1999, 16, 388-394. [CrossRef] [PubMed] 
46. Philibert, D.; Cattran, D. Remission of proteinuria in primary glomerulonephritis: We know the goal but do we know the price? Nat. Clin. Pract. Nephrol. 2008, 4, 550-559. [CrossRef]

47. Shihabi, Z.K.; Konen, J.C.; O'Connor, M.L. Albuminuria vs urinary total protein for detecting chronic renal disorders. Clin. Chem. 1991, 37, 621-624. [CrossRef]

48. Hruskova, Z.; Honsova, E.; Berden, A.E.; Rychlik, I.; Lanska, V.; Zabka, J.; Bajema, I.M.; Tesar, V. Repeat protocol renal biopsy in ANCA-associated renal vasculitis. Nephrol. Dial. Transplant. 2014, 29, 1728-1732. [CrossRef]

49. Xu, P.C.; Chen, T.; Gao, S.; Hu, S.Y.; Wei, L.; Yan, T.K. Clinical and pathologic characteristics of pauci-immune anti-myeloperoxidase antibody associated glomerulonephritis with nephrotic range proteinuria. Ren. Fail. 2018, 40, 554-560. [CrossRef] 